\title{
Carnets
}

Revue électronique d'études françaises de l'APEF

Première Série - 3 Numéro Spécial | 2011

La littérature face au «politiquement correct». Notions, pratiques et dérives

\section{Christine Angot: le desir d'indigner le lecteur : La société violée par l'(auto)fiction}

\section{Francesca Forcolin}

\section{(2) OpenEdition}

Journals

\section{Édition électronique}

URL : http://journals.openedition.org/carnets/6374

DOI : $10.4000 /$ carnets. 6374

ISSN : 1646-7698

Éditeur

APEF

Édition imprimée

Date de publication : 1 juin 2011

Pagination : 51-61

\section{Référence électronique}

Francesca Forcolin, «Christine Angot: le desir d'indigner le lecteur : La société violée par l'(auto)fiction », Carnets [En ligne], Première Série - 3 Numéro Spécial | 2011, mis en ligne le 19 juin 2018, consulté le 01 mai 2019. URL : http://journals.openedition.org/carnets/6374 ; DOI : 10.4000/carnets.6374

\section{(c) (i) (8)}

Carnets est mis à disposition selon les termes de la licence Creative Commons - Atribution - Pas d'utilisation commerciale 4.0 International. 


\title{
CHRISTINE ANGOT: LE DESIR D'INDIGNER LE LECTEUR La société violée par l'(auto)fiction
}

FRANCESCA FORCOLIN

Université de Turin - Université de Nantes

francesca.forcolin@gmail.com

\begin{abstract}
Résumé
Christine Angot, "reine de l'autofiction", met sur la page ses pensées les plus intimes, les expériences extrêmes de son double de papier, à travers une écriture ambiguë qui se contredit et s'annule. En se déclarant homosexuelle et puis homophobe, elle a des propos offensifs, qui font de son écriture un geste d'irrévérence sociale. Le lecteur est ainsi obligé de se poser des questions, sur la notion de "politiquement correct", sur la société et ses tabous. Nous montrerons que la volonté de provoquer, afin de déclencher une réponse, peut être considérée comme une sorte d'“engagement" postmoderne. Le lectorat, indigné, est obligé de réfléchir sur ses préjugés et de les combattre, même si cela comporte pour l'auteur le risque d'un rejet définitif.
\end{abstract}

\begin{abstract}
Christine Angot, "queen of autofiction", writes down her most intimate thoughts. Her writing has always been characterised by the ambiguity of its context, renowned for an abundance of contradictions. By declaring herself homosexual and homophobe, her injurious confessions made her writing an example of social irreverence. The reader is obliged to think again and reflect on what society and its restraints consider to be "politically correct". We will show how her will to shock in order to have a reaction could be considered a kind of post-modernism "engagement" of the writer. The reader is obliged to think about his own prejudices and to fight them, even if there is for the author the risk of a definitive reject.
\end{abstract}

Mots-clés: Angot, autofiction, engagement, tabous, inceste, homosexualité

Keywords: Angot, autofiction, engagement, taboos, incest, homosexuality 
Christine Angot, "la bâtarde libre" (Guichard, 1997: 18), place son écriture sous le signe de la transgression, de l'insolence. Le but est faire parler de soi, avoir une réponse soit-elle positive ou négative - de la part des lecteurs. En partant de l'hypothèse de ce "politiquement incorrect", j'examinerai en particulier deux de ses romans - les romans-pivots - le premier de 1993, Léonore, toujours, le deuxième de 1999, L'Inceste, romans où la présence envahissante, proliférante du je de l'auteure nous semble un aveu narcissique de son omniprésent double, "Christine".

La volonté est de bouleverser ce qu'il faut dire, ce que la société nous a imposé de dire, la volonté de détruire de l'intérieur le "politiquement correct", éliminant exprès toute attention verbale envers les catégories mineures - par exemple sexuelles. C'est un "politiquement incorrect" qui se traduit par la volonté médiatique de créer le scandale à travers le manque d'autocensure, à travers une écriture parfois érotique, parfois androgyne, qui met au centre - en les attaquant - les tabous de la société.

Subvertir donc les canons, aller contre le bien-dit, les speech codes, contre, on pourrait dire, l'hypocrisie sociale ou la cosmétologie verbale - comme dirait Umberto Eco, qui voit dans le "politiquement correct" une solution outrageuse qui change le nom mais ne résout pas le problème ${ }^{1}-$, utilisant donc des formes verbales qui gênent et font trembler les paladins (ou bien la société entière) des bonnes normes, afin que cette insolence, en troublant, puisse déclencher une réponse. Attentat au bon goût ou énonciation sans censure de pensées que partagent la plupart des gens, mais qui restent intimement cachées sous les conventions sociales?

Pour cette analyse, les axes méthodologiques seront deux: en premier lieu, il sera question de la contradiction, ou jeu d'opposition entre réalité et fiction; deuxièmement, de la réponse du public - dans la double déclinaison de l'acceptation et du refus.

Le point de départ de notre analyse est la déclaration faite en 1993, dans le roman Léonore, toujours, où Angot, en se référant à sa fille, affirme:

Mais si j'en fais une homosexuelle, quel échec! Quel dommage! Si on me donne le choix entre violée et lesbienne, je prends le viol. Violée, les déchirures se succèdent. Anales, vaginales, tout y passe, la bouche, le sillon des seins. [...] Je suis une mère normale qui veut une fille normale (Angot, 1993: 37).

Une déclaration où les notions clés à examiner sont celles de: homosexualité, viol et normalité.

"She is renowed for making statements that are not politically correct" (Rye, 2004:

\footnotetext{
1 Umberto Eco traite la complexité de la notion de "politiquement correct" dans l'article intitulé "Pistola dell'Ostrega", paru le 1 juillet 2004 dans l'hebdomadaire L'Espresso. II y analyse brièvement l'histoire du mot, ses contradictions et l'importance de la liberté linguistique.
} 
123), affirme le critique Gill Rye. Dans Léonore, toujours, qui a la forme d'un journal relatant les événements du lundi 8 mars au mercredi 31 mars, la narratrice parle de son bébé Léonore de huit mois et imagine toutes sortes de futurs possibles pour elle. Angot décrit son rapport avec sa fille qui vient de naître - un rapport ambigu très proche de celui décrit par Marie Darrieussecq dans Le mal de mer (Darrieussecq, 1999) - en insistant sur l'ambivalence des sentiments, les joies et les douleurs, mais surtout sur la difficulté à gérer maternité et écriture. Mais il est impossible de ne pas penser qu'il s'agit d'un rapport ayant une veine morbide. Angot écrit: "Je ne veux pas faire d'inceste avec elle physiquement. Mais dans la tête, ce n'est pas possible autrement" (Angot, 1993: 11).

Angot mêle à la manière d'un stream of consciousness joycien ses pensées et ses fantaisies - fantaisies sexuelles, désirs, idées qui dans l'esprit occupent l'espace d'un instant, flash intimes qui montrent la jouissance maternelle, selon les mots de Julia Kristeva, un désir souvent caché, un élément émanant d'un ordre social patriarcal. C'est un rapport mère-fille sous le signe de la transgression; Léonore, toujours est loin d'être un texte doucereux, au contraire, Angot dit ce qu'il ne faut pas dire, ce qui est considéré inadmissible.

La question de l'inceste est la première contradiction sur laquelle nous butons. En effet, l'inceste occupe une place centrale dans l'écriture d'Angot, c'est le fil rouge de tous ses romans. L'inceste avec son père - la présence obsessive du père dans son écriture nous porte à parler d'une sorte de "récit de filiation"² dans le sillage de Dominique Viart - occupe en particulier le dernier chapitre du roman homonyme, inceste qui a eu lieu pendant deux ans, de ses quatorze à ses seize ans. A partir de cette expérience naît chez Christine la culpabilité, pour avoir éprouvé un plaisir physique qu'elle ne cache jamais, mais en même temps se manifeste chez elle la nécessité d'utiliser l'écriture comme instrument thérapeutique. Elle écrit: "Prendre le pouvoir, avoir le dessus. Et maintenant, je l'ai. Moi j'ai le dessus sur l'inceste. Le pouvoir, le pénis sadique, ça y est, grâce au stylo dans la main sûrement, essentiellement" (Angot, 1999: 152).

Peut-on préférer le viol, l'inceste, à l'homosexualité? Peut-on accepter que l'écrivaine même qui dit avoir vécu ce trauma puisse affirmer qu'elle choisirait un tel futur pour sa fille? Ici, le lecteur se heurte à la deuxième contradiction.

En effet, au début de L'Inceste, Angot se déclare homosexuelle. Elle écrit: "J'ai été homosexuelle pendant trois mois. Plus exactement, trois mois, j'ai cru que j'y étais condamnée. J'étais réellement atteinte, je ne me faisais pas d'illusions" (idem: 11).

Tout le long du texte, Christine fait allusion à cette prétendue homosexualité en

\footnotetext{
2 "Cette forme littéraire a pour originalité de substituer au récit plus ou moins chronologique de soi qu'autofiction et autobiographie ont en partage, une enquête sur l'ascendance du sujet. [...] Les écrivains remplaçaient l'investigation de leur intériorité par celle de leur antériorité familiale. Père, mère, aïeux plus éloignés, y sont les objets d'une recherche dont sans doute l'un des enjeux ultimes est une meilleure connaissance du narrateur de lui-même à travers ce(ux) dont il hérite" (Viart, 2009: 96).
} 
parlant de son rapport lesbien avec le médecin Marie-Christine, une femme plus âgée qu'elle, et elle déclare même que l'homosexualité l'a toujours fascinée, bien qu'elle ait un mari, Claude, et une fille. La passion physique est décrite sans détours: "Pour faire la chose que je ne fais jamais, lécher je peux le dire, j'espérais en être dégoutée à jamais. Elle n'a pas voulu du tout qu'on fasse l'amour. [...] C'est la vie mais ça réagissait, quand elle me léchait. Positif." (idem: 22). Toujours dans L'Inceste, au début du deuxième chapitre, "Noël”, Angot définit l'homosexualité en citant le Dictionnaire de la psychanalyse d'Elisabeth Roudinesco et Michel Plon. Voilà ce qu'on y dit de l'homosexualité:

Freud conserva le terme perversion pour désigner des comportements sexuels déviants par rapport à une norme structurale (et non plus sociale) et il rangea I'homosexualité. [...] Elle a pour origine chez la fille une fixation infantile à la mère et une déception à l'égard du père. [...] II n'y a là rien dont on doive avoir honte, ce n'est ni un vice, ni un avilissement et on ne saurait la qualifier de maladie; nous la considérons comme une variation de la fonction sexuelle, provoquée par un arrêt du développement sexuel. [...] C'est une grande injustice de persécuter l'homosexualité comme un crime, et c'est aussi une cruauté. [...] L'homosexualité dans sa version féminine comme une identification à un pénis sadique (idem: 118-119).

Cette "variation", nommée aussi "perversion" (idem: 119), est souvent associée au nazisme: "Le nazisme, je persécute Marie-Christine parce qu'elle est homosexuelle, alors que c'est juste une variation, provoquée par un arrêt du développement sexuel" (idem: 129).

Dans ces définitions Angot utilise le langage freudien pour donner une explication clinique et médicale de l'homosexualité, pour convaincre les gens à ne pas marginaliser ceux qui, dans la plupart des cas, ne sont pas considérés comme des personnes "normales". Et pour insister sur sa prétendue homosexualité, Angot nous parle des difficultés que rencontrent gays et lesbiennes dans la vie quotidienne, des situations problématiques qu'elle dit vouloir vivre au cours de la relation avec son partenaire, et elle en parle comme d'une différente forme d'amour: "L'appartement, les PACS, tout ce qui est à elle que ça me revienne. [...] Je rêve: nous avons une maison. [...] Léonore est là. Personne ne peut rien trouver à redire" (idem: 23 et 42).

Ici, Angot est donc très proche des homosexuels en se déclarant telle, et en utilisant aussi des expressions qui ne sont pas blessantes pour le lecteur. Mais comment peut-on expliquer ce changement radical, cette prise de position qui, d'un texte à l'autre, semblent même être prononcés par deux auteurs différents? A-t-on devant soi toujours la même Christine? Et encore: est-ce qu'on peut considérer la déclaration d'homosexualité comme un véritable aveu dans la page fictive du texte littéraire?

Dans le premier chapitre de L'Inceste, la déclaration d'homosexualité est un calque 
de l'incipit de A l'ami qui ne m'a pas sauvé la vie d'Hervé Guibert, "autobiographie homosexuelle" publiée en 1990, où l'auteur évoque son combat contre le sida, dévoilant aussi les circonstances de la mort de Michel Foucault, qui y figure sous le pseudonyme du professeur Muzil. Guibert écrit: “J'ai eu le sida pendant trois mois. Plus exactement, j'ai cru pendant trois mois que j'étais condamné par cette maladie mortelle qu'on appelle sida" (Guibert, 1990: 9).

On voit donc la stricte analogie avec le roman d'Angot, qui reprend ce texte en remplaçant la maladie par l'homosexualité, en se déclarant "atteinte" comme Guibert par le virus. Même si les passages sont réécrits sans guillemets ou notes, on est loin de pouvoir considérer ces emprunts comme un plagiat, un vol, il s'agit plutôt d'un hommage à un écrivain à qui Angot doit beaucoup. En effet, le jeu intertextuel est explicite, il est bien mis en évidence par Angot qui est définie par le critique Demoulin comme "la petite sœur d'Hervé Guibert" (Demoulin, 2002: 643). Elle utilise toutes les pistes à sa disposition pour rendre compréhensible sa démarche: ces emprunts sont pour la plupart détachés du contexte; ils comportent des éléments hétérogènes qui les démasquent, et sont situés à un endroit très visible, comme l'aveu d'homosexualité en tête de page. L'écriture de Guibert est un modèle pour Angot, et elle le déclare explicitement dans Léonore, toujours: "Je veux faire exactement comme Hervé Guibert avec le sida" (Angot, 1993: 17).

A travers l'emprunt guibertien, et si l'on prend pour vrai le jeu fictif du texte, on peut supposer que l'homosexualité d'Angot durera plus que trois mois. En effet à partir du titre même du roman de Guibert, on comprend qu'il ne sera pas sauvé par son "ami", le médecin chercheur, et que son sida durera au-delà des trois mois. De surcroît, on sait que Guibert est mort des suites de la maladie. On peut donc supposer qu'Angot adopte le même processus de "dénégation désespérée" (Demoulin, 2002: 642), un jeu d'aveu et dénégation. Aveu masqué de citation, et aveu dénié d'homosexualité: un rapport lesbien de courte durée qui, suivant l'analogie avec Guibert, perdure au-delà des trois mois, mais qui peut être considéré un désir momentané imprimé dans la fantaisie de l'auteure qui n'est jamais devenu un acte réel.

Comme tous les livres d'Angot font appel à d'autres textes, l'aveu dans L'Inceste peut être considéré un simple jeu intertextuel, une volonté de mettre en évidence la logique incestueuse de son écriture. En effet, dans son œuvre, on trouve des références à d'autres écrivains (Marguerite Duras, Jean-Jacques Rousseau, Marcel Proust, Marina Tsvetaieva, Camille Laurens), à la Bible, au cinéma (Jean Eustache), mais aussi des véritables réécritures, comme le roman Peau d'âne, à partir du conte homonyme de Perrault. A ce propos, Angot déclare: "Souvent, j'utilise des livres pour écrire" (Angot, 1998c: 24). Elle exprime avec violence son homosexualité, mais d'autres déclarations portent le lecteur à ne pas croire aux tendances lesbiennes de l'auteure. En se référant à sa fille, elle dit: 
Je suis hétérosexuelle. Ma chérie. Bien sur. Hétéro. Comment j'aurais eu sinon une si jolie fille? Jamais, tu m'entends bien, jamais, je n'avais eu de désir pour une femme. Le sexe de l'homme pénètre de façon radicale. J'aime bien ce qui est radical (Angot, 1999: 43).

Nous avons donc l'impression très forte qu'il s'agisse d'un jeu de citations, de cachecache, mais surtout d'un moyen pour mettre mal à l'aise le lecteur, une volonté de créer scandale en mettant sur la page, sans pudeur, un amour lesbien. Est-ce que nous lisons I'histoire d'un amour à traits pornographiques d'un être de papier nommé Christine, ou bien un aveu de l'écrivaine même, qui se sert des mots de Guibert pour pousser plus loin la confusion du lecteur?

Depuis toujours connue comme une écrivaine qui "fait scandale" pour la "merde de témoignage" (idem: 198) qu'elle donne en superposant, dans ses romans, auteur, narrateur et personnage et en laissant le lecteur toujours dans le doute, Christine Angot a été consacrée, bien que contre son gré, romancière autofictive, à l'occasion du roman L'Inceste qui l'a faite sortir de l'ombre, et après son passage à l'émission de Bernard Pivot le 3 septembre 1999 - un Bouillon de culture mémorable, où elle décrit avec véhémence sa position par rapport à la littérature. Parmi les autres, cette étiquette lui a été imposée par Vincent Colonna, qui la cite parmi les auteurs francophones "pratiquant une forme ou l'autre de fiction de soi" (Colonna, 2004: 195), avec Marguerite Duras, Serge Doubrovsky, Annie Ernaux, Michel Houellebecq, auteurs qui font "un type de roman dans lequel le personnage principal porte le nom de l'auteur et où règne la vraisemblance biographique" (idem: 196). Une volonté d'extimité, donc, une vague de dévoilement de l'intime, dans une époque hautement vouée à l'individualisme.

Dans l'écriture angotienne haletante, saccadée, orale, où la syntaxe est hachée et décousue, les phrases nominales qui se contredisent et s'annulent portent le doute au paroxysme. Doute qui nait de la difficulté à distinguer personnages réels et fictifs, la Christine vraie et celle de papier, dans des romans proches du journal intime qui imbriquent, à travers un jeu sadique, vie et littérature. La confusion vertigineuse qui règne mime le même désarroi de l'écrivaine, qui, en parlant toujours d'elle même (à tel point que Christophe Donner a pu définir son écriture d' "angotcentrique". (Faerber apud Donner, 2002: 48)), n'arrive en réalité jamais à se connaître: “J'ai l'impression de parler de quelqu'un d'autre” (Angot, 1999: 24).

Et la rhétorique de l'altérité est centrale: l'ego d'Angot, en effet, ne s'éprouve jamais un, mais dit la pluralité, et cet échec d'unité ontologique conduit à une aliénation par rapport à soi qui prend souvent la forme de la folie. Le je est toujours en proie au questionnement identitaire (le "Qui suis-je?" d'André Breton (Breton, 1963: 9)), et il est perpétuellement à la 
recherche de soi. Le je devient toujours autre, et il se décrit à travers les voix des personnes de sa famille ou de ses amis, comme dans Sujet Angot, roman de 1998, où l'auteure parle de soi à travers son mari Claude, à la manière de The autobiography of Alice Toklas de Gertrude Stein (Stein, 1933).

Dans cette continuelle recherche de soi, dans ce jeu entre réalité et fiction (à ce propos, Guichard affirme: "Il faut que le lecteur soit dans le doute. Dans la littérature, on part avec l'idée que tout est mensonge et une fois plongé dans le livre, on prend tout pour acquis" (Rye apud Guichard, 2004: 117) l'auteure tourne autour de sa personne ("c'est sa monotonie l'unique matière de son écriture" (Colonna, 2004: 115)), l'auteure exprime ses goûts, ses désirs, ses qualités et défauts, ses faiblesses et son intimité, à travers une écriture du corps qui est à la fois impudique et audacieuse, érotique, et où les caractères sexuels sont brouillés.

Cette écriture androgyne ${ }^{3}$ gêne la lecture, c'est une écriture extrême qui parfois nous intimide (ce n'est pas hors propos de citer le sentiment de Marguerite Duras au sujet de l'œuvre bataillienne, œuvre capitale qui a eu une très grande influence sur celle d'Angot ${ }^{4}$ : "les gens continuent à vivre dans l'illusion qu'ils pourront un jour parler de Bataille. Ils mourront sans oser, dans le souci extrême où ils sont de leur réputation, affronter ce taureau" (Duras, 1958: 34)).

Mais le point central est le dépaysement du lecteur, obligé de se confronter à luimême et à la société entière. Voyons pourquoi.

Les déclarations faites dans Léonore, toujours se situent très loin du respect envers les minorités et suscitent un trouble immédiat. Cependant, selon Angot l'écrivain a la "liberté" de tout dire. L'écriture est un acte fondateur, une libération, et c'est à travers l'écriture qu'on affirme l'indépendance absolue de l'individu. L'écrivain a donc le droit de tout écrire, à condition que ce soit une vérité partielle. Dans L'usage de la vie, essai théorique de 1998, elle écrit: "Les écrivains ne devraient jamais cesser d'écrire leur vie en fait. Avec le doute, qui plane. Sur la vérité" (Angot, 1998b: 10). L'écrivain écrit ce qu'il ressent, le déforme; il a les droits de tout dire, même si ça vexe, même si ça choque.

Et la volonté d'Angot est décidément celle de choquer. Comme on a vu, elle décrit son rapport lesbien sans pudeur. Elle affirme avec violence la volonté d'avoir une fille normale, c'est à dire hétérosexuelle, en soulignant ainsi l'anormalité de l'homosexualité.

La volonté de l'auteure est celle de montrer la violence de la société, du "drap social"

\footnotetext{
${ }^{3}$ Ou bien "Angotgyne", la définition de Johan Faerber, selon qui l'écriture angotienne est une continuelle recherche de la partie de soi manquante, une tentative de réconciliation des contraires et des notions de masculin et de féminin, qui va s'effectuer dans la relation de Christine avec Marie-Christine de L'Inceste: "Pour Angot, c'est l'écriture qui, seule, apporte l'Amour, réconcilie et soude les antithèses" (Faerber, 2002: 58).

${ }^{4} \mathrm{~A}$ ce propos, il faut souligner, entre autres, le récit d'Angot, "Chère Madame", paru dans la revue L'Infini (Angot, 1998a: 21). Ici, le rapport avec l'œuvre bataillienne est très explicite, puisque c'est le sujet (caché à travers un jeu d'aveu et dénégation) du récit même.
} 
comme elle définit le monde. Elle le fait à travers les expériences propres à Christine (l'être de papier), une sorte de mise en abyme qui reproduit et amplifie cette violence pour la donner à entendre.

La violence envers le lecteur est le moyen pour lui faire tomber le masque et l'obliger à prendre position. Gill Rye affirme: "In precisely linking heterosexuality, normality, and the expression of a preference for rape over lesbian sex, despite Christine's experiences, Angot confronts her readers with their own prejudices and challenges them to think again" (Rye, 2004: 124).

La vie de Christine est donc un moyen pour obliger le lecteur de se confronter au monde et à ses idées, de reconnaître ses propres préjugés et de les combattre. On pourrait presque parler, même si c'est peut-être hasardé, d'une sorte d'“engagement" de la part de Christine Angot, qui ne peut bien sûr plus avoir ni le fond ni la forme de celui sartrien, qui n'a pas une forme encore bien définie, mais qui est tout de même un engagement post-moderne dont les confins sont brouillés. Le lecteur est obligé de faire sortir le sens de l'œuvre, de comprendre la démarche du texte et ses significations, et la difficulté réside dans le manque d'une linéarité et de cohérence textuelle, mais aussi - et surtout - dans la confrontation à la société, qui, selon le critique Eva Domeneghini, "est nécessairement violente, et la confrontation de l'écrivain au public ou au monde de l'édition sert en quelque sorte de métaphore métonymique à une démonstration qui se veut universelle" (Domeneghini, 2002). Un engagement envers la société et envers soi-même, ses propres tabous, pudeurs, une lutte qui a été considérée "périmée" - de la même manière que les notions de personnage, histoire, forme et contenu, selon les Nouveaux Romanciers: "[...] dès qu'apparait le souci de signifier quelque chose (quelque chose d'extérieur à l'art) la littérature commence à reculer, à disparaître" (Robbe-Grillet, 1961: 39).

La vie toute entière et ses tabous sont donc au cœur de l'œuvre angotienne. Elle traite des sujets pénibles, sujets omniprésents dans la société actuelle, comme l'inceste, le sida, I'homosexualité, la crise du couple, la violence dans les relations amoureuses.

Habituée à tourner le regard de l'indicible, du non-dit, de l'horreur et de la violence souvent cachée, la société considère cette écriture offensive et amorale puisqu'elle dévoile le plus intime. A ce propos, en citant l'essai de Philippe Forest, Le roman, le réel: un roman est-il encore possible? (Forest, 1999), on peut envisager cet indicible comme un reste. Forest fait l'exemple mathématique 10:3. Le plus loin qu'on aille après la virgule, on a toujours un reste. Ce reste, dit Forest, est ce que la société refuse, le non-dit toujours caché, ce qui fait horreur, comme une jeune fille violée par son père ou les souffrances d'un enfant.

Angot met en scène son corps, le sexe, avec un exhibitionnisme qui va au-delà de l'écriture intime, où le lecteur n'est plus un confident - comme dans l'autobiographie classique - mais devient souvent un voyeur. Mais comme elle expose sans pudeur sa vie 
intime et empêche qu'on puisse comprendre où sont les limites de la fiction, les lecteurs peuvent réagir en refusant avec indignation ces documents, ces confessions, et en exigeant le rétablissement d'une différenciation saisissable entre ce qui a été vécu et ce qui est fictif.

C'est pour cette raison qu'Angot a été accusée à plusieurs reprises par ses détracteurs de nombrilisme et de narcissisme ${ }^{5}$, dans une écriture qui se fait vie et une vie qui se fait écriture. Elle expose sa vie et ses amours, hétéro ou gays, dans les émissions télévisuelles et dans le texte littéraire.

L'apparition de L'Inceste a suscité une tourmente médiatique qui a divisé les lecteurs, entre ceux qui ont ressenti un rejet définitif, et ceux qui ont éprouvé une admiration soudaine pour l'œuvre de l'écrivaine. Entre autres, il faut citer Philippe Sollers qui affirme, dans Le Journal du Dimanche, à propos de Rendez-vous publié en 2006:

Excellent, puissant, rapide, audacieux, drôle. Vous commencez à lire, c'est immédiat, vous ne lâchez plus les pages, vous vous demandez comment elle va se tirer d'une folie parfaitement maîtrisée, inceste traumatique, sincérité, crudité lucide. Eh oui, il faut s'y faire: Angot est un des meilleurs écrivains français d'aujourd'hui. But marqué, donc. C'est rare (Sollers, 2006).

Les détracteurs ne manquent pas, et la critique l'a accusée aussi d'atteinte à la vie privée, puisque les noms des personnages de ses romans sont ceux des personnes réelles ("Tout le manuscrit pose un problème global d'atteinte à la vie privée des personnes qui y sont mentionnées, décrites" (Angot, 1999: 38)). C'est "la soif contemporaine de réel" (Crépu, 2001), la tendance à incorporer dans les romans des morceaux entiers du réel, ce qui mène parfois à des poursuites judiciaires, comme c'est arrivé également à Michel Houellebecq pour Les particules élémentaires (Houellebecq, 1998).

Le public, menacé par cette exposition de l'intime, est pris à témoin et devient le juge, "mes lecteurs sont mes sauveurs" (Angot, 1999: 74). On pourrait même dire qu'il est incorporé dans l'œuvre d'Angot, au point qu'il trouve parmi les personnages son alter ego, comme le mari Claude, qui imposant à Christine de ne pas formuler des jugements trop rapides, lui donne voix. On peut le voir dans Léonore, toujours: Christine recopie des phrases du carnet de Claude à propos de la beauté de se trouver dans les bras d'une femme. Elle réagit disant: "Alors que notre ami Patrick, homosexuel, à la veille de sa mort, était encore un pauvre type"; il suit, entre parenthèses, la réprimande de Claude-lecteur: "(Claude va encore me dire 'tu te rends compte de ce que tu écris?')" (Angot, 1993: 125).

Une mise en abyme du lecteur, ce qui nous rappelle que l'écriture d'Angot "is always

\footnotetext{
${ }^{5}$ Angot défend la littérature française attaquée injustement: "Qu'on arrête de dire que la littérature française c'est fini. Narcissique, nombrilique" (Angot, 1998b: 9).
} 
in question, and always to be questioned" (Rye, 2004: 124). Encore une fois, donc, la mise en abyme, figure fictionnelle très proche de l'autofiction, selon Colonna, qui écrit:

En mettant en circulation son nom, dans les pages d'un livre dont il est déjà le signataire, l'écrivain provoque, qu'il le veuille ou non, un phénomène de redoublement, un reflet du livre sur lui-même ou une monstration de l'acte créatif qui l'a fait naître (Colonna, 2004: 132).

Le contact physique entre lecteur et écrivain est donc très fort, un lecteur qui est à la fois juge et Autre, et à travers lequel Angot se décrit. On est toujours dans l'entre-deux, un passage qui conduit d'une intimité à l'autre, de celle de l'écrivaine à celle du lecteur, qui est invité, toujours avec violence, à considérer si certains jugements sont acceptables ou ne le sont pas, et si ses propres pensées intimes sont, effectivement, "politiquement correctes". "Comme un ver bien installé dans le fruit de notre société" (Guichard, 1997), Angot dévoile la pensée intime d'une grande partie de la société, en créant confusion et scandale, indignation des communautés homo et hétéro.

Pour conclure, nous pensons que le "politiquement incorrect" d'Angot peut être considéré une sorte d'“engagement" post-moderne, comme nous l'avons suggéré plus haut, et que le systématique brouillage des pistes la mène à une écriture où la frontière entre réel et fictif est très mince, au point qu'il est presque impossible de distinguer la voix de l'auteure de celle du personnage fictif - ce qui nous pousse à croire que l'aveu d'homosexualité d'une part et, d'autre part, le jugement homophobe, exprimés dans Léonore, toujours, sont, l'un et l'autre, des vérités purement textuelles.

Dans cette écriture de provocation, il y a, simultanément, une recherche de l'unité ontologique perdue à l'âge d'or de l'enfance et le refus d'une image univoque de sa personne, de la part d'Angot; cela mène à la naissance des nombreuses voix d'une auteure qui n'arrive jamais à se définir. La pluralité du je s'exprime à travers ces voix discordantes, ces voix des nombreux personnages qui portent le même nom que l'auteure; il s'agit alors d'un jeu d'ambiguïté extrême, profondément marqué par la présence de déclarations contradictoires et par le manque de cohésion identitaire. Quoi qu'elle en dise (dans le sillage de Robbe-Grillet, "Je ne parle que de moi" (Angot, 1993: 14)), la fiction est le moteur propulsif de son écriture.

Un engagement, donc, puisqu'elle pointe son doigt vers les tabous de la société, vers ce qui est obscène et ce qui n'est pas admis en s'y plongeant, même si cette provocation, obligeant le lecteur à réagir, comporte le risque d'un rejet définitif: "The state of not-knowing in Angot's work may operate as an impetus for the reader to think again - and thus, paradoxically, to know better" (Rye, 2004: 125). 


\section{Bibliographie}

ANGOT, Christine (1993). Léonore, toujours. Paris: Fiction \& Cie.

ANGOT, Christine (1998). Sujet Angot. Paris: Fayard.

ANGOT, Christine (1998a). "Chère Madame". In: L'Infini, n64, pp. 21 -30.

ANGOT, Christine (1998b). L'usage de la vie. Paris: Fayard.

Angot, Christine (1999). L'Inceste. Paris: Stock.

Angot, Christine (2003). Peau d'âne. Paris: Stock.

ANGOT, Christine (2006). Rendez-vous. Paris: Gallimard.

BRETON, André (1963). Nadja. Paris: Gallimard.

COLONNA, Vincent (2004). Autofiction et autres mythomanies littéraires. Paris: Tristram.

CREPU, Michel (2001). "Le roman français est-il mort?" [on-line]. In: L'Express [consulté le 1/12/2010] <URL: lexpress.fr/informations/le-roman-francais-est-il-mort_641861.html>

DARRIEUSSECQ, Marie (1999). Le mal de mer. Paris: POL.

Demoulin, Laurent (2002). "Angot salue Guibert”. In: Critique, vol. LVIII, n663, pp. 638 -644.

DEN TOONDER, Jeanette (2005). "L'autoreprésentation dans une époque massmédiatisée: le cas Angot”. In: Sjef Houppermans, Christine Bosman Delzonz, Danièle de Runter-Tognotti (éds.). Territoires et terres d'histoires - Perspectives, horizons jardins secrets dans la littérature française d'aujourd'hui. Amsterdam-New York: Rodopi, pp. 39-60.

DOMENEGHINI, Eva (2002). "Impressions sur l'œuvre de Christine Angot” [on-line]. In: La revue des ressources [disponible le 1/12/2010] <URL: larevuedesressources.org/spip.php?article77>

DURAS, Marguerite (1958). "A propos de Georges Bataille". In: Hommage à Georges Bataille, La Ciguë, n9, pp. $32-33$.

Eco, Umberto (2004). "La pistola dell'ostrega” [on-line]. In: L'Espresso [consulté le 15 décembre 2010] $<U R L$ : bekar.net/ospiti/giornalisti/docs/art/pol_corretto.rtf>

FAERBER, Johan (2002). "Le bruissement d'elles, ou le questionnement identitaire dans l'œuvre de Christine Angot". In: Nathalie Morello, Catherine Rodgers (orgs). Nouvelles écrivaines: nouvelle voix?. New York: Rodopi, pp. 47-62.

FOREST, Philippe (1999). Le roman, le réel - un roman est-il encore possible? Nantes: Pleins Feux.

GUIBERT, Hervé (1990). A l'ami qui ne m'a pas sauvé la vie. Paris: Gallimard.

GUICHARD, Thierry (1997). "Christine Angot, la bâtarde libre". In: Le matricule des anges, n21, pp. 18 19.

HOUELLEBECQ, Michel (1998). Les particules élémentaires. Paris: Flammarion.

Robbe-Grillet, Alain (1963). Pour un Nouveau Roman. Paris: Minuit.

RYE, Gill (2004). "Il faut que le lecteur soit dans le doute: Christine Angot's literature of uncertainty". In: Dalhousie French Studies, vol.68, pp. 117-126.

RYE, Gill (2005). "In uncertain terms: mothering without guilt in Marie Daurrieussecq's 'Le mal de mer' and Christine Angot's 'Léonore, toujours'”. In: L'esprit créateur, vol.XLV, n9, pp. 5-15.

STEIN, Gertrude (1933). The autobiography of Alice Toklas. New York: Literary guild.

SOLLERS, Philippe (2006). "L'actualité vue par Sollers - Angot" [on-line]. In: Le journal du mois [disponible le 17/01/2011] <URL: pileface.com/sollers/article.php3?id_article=196>

VIART, Dominique (2009). "Le silence des pères au principe du récit de filiation". In: Figures de l'héritier dans le roman contemporain, Etudes françaises, 45,3. Montréal: Les presses de l'Université de Montréal. 
